Vol.12,\#8, Junio 2007

http://revista-redes. rediris.es

\title{
La formación de redes sociales en el estudio de actores y familias. Perspectiva de estudio en historia y antropología
}

\author{
Carmen I melda González Gómez y Manuel Basaldúa Hernández - \\ Universidad Autónoma de Querétaro ${ }^{1}$
}

\begin{abstract}
Resumen
La noción de redes sociales se puede elevar a modelo de estudio cuando se aplica para mostrar y explicar el entramado de relaciones entre diversos individuos y grupos en su interacción social dentro de un sistema o estructura. La sutileza del uso del concepto de redes sociales puede ser observada en la descripción del proceso de momentos clave de las relaciones de los actores en su trayectoria histórica, así como en la funcionalidad que cada uno ellos expanden en la estructura social a la que pertenece. Estos términos y categorías permiten plantear que el principio de redes sociales puede ser utilizado desde distintas perspectivas teóricas y disciplinas, como en el caso de la historia y la antropología.

Los autores plantean una postura teórica dirigida al estudio particular de sus casos abordados, pero encuentran una convergencia metodológica en la explicación de los procesos de formación de redes sociales. El descubrimiento de estos elementos sale a la luz después de analizar los datos en épocas y escenarios diferentes, pero comunes en los patrones de las relaciones de vinculación social de los gremios o sectores específicos. También, con la mirada de sus respectivas disciplinas exploran y comparan los mecanismos sociales como un ejercicio transdisciplinario. El propósito es profundizar en ese carácter interdisciplinario del estudio de las redes sociales a partir de protagonistas y escenarios distintos.
\end{abstract}

Palabras clave: redes sociales - relaciones personalizadas - vínculos familiares vínculos de negocios.

\begin{abstract}
The notion of social networks can be raised to a study model when it is applied to show and explain the intertwining relations among diverse individuals and groups in their social interaction inside a system or structure. The subtlety of the use of the concept of social networks can be observed in the description of key moments in the relations of the actors in their historic trajectory, as well as in the functionality that each of them expand in the social structure where they belong. These terms and categories let us establish that the beginning of social networks can be used from different theoretical perspectives and disciplines, two of these being history and anthropology.

The authors state a theoretical posture directed to the particular study of the discussed cases, but find a methodological convergence point in the explanations of the processes of formation of the social networks. The discovery of these elements is brought to light after analyzing the data in different times and scenarios, but communes in the patterns of social connections among the guilds or specific sectors. Also, from an overview of each of their disciplines they explore and compare the social mechanisms in an interdisciplinary exercise. The purpose is to look further into this

\footnotetext{
${ }^{1}$ Profesores-Investigadores de la Facultad de Ciencias Políticas y Sociales. Universidad Autónoma de Querétaro, Cerro de las Campanas, Av. Hidalgo s/n, Centro Histórico. México. C. P. 76000 carmenigonzalez@hotmail.com y basaldua@uaq.mx.
} 
interdisciplinary study of the social networks parting from diverse characters and scenarios.

Key words: social networks - custom-made relations - family bonds - business bonds.

\begin{abstract}
"Hablamos en suma de redes, no meramente agentes individuales... Sólo así, por lo demás podemos tener una visión de conjunto con independencia de que desconozcamos algunos mecanismos particulares. Aunque cada individuo busque la maximización individual, ésta solo la obtiene con la colaboración de otros, y así se genera la red, que luego se superpone como hecho social al individuo - lo mismo podríamos decir en términos de grupos, de clases sociales incluso-.
\end{abstract}

Artemio Baigorri, Hacia la urbe global, ¿El fin de las jerarquías territoriales?, Universidad de Extremadura, julio, 1998.

\title{
I ntroducción
}

El propósito de este trabajo es articular dos disciplinas sociales para mostrar que las redes sociales se convierten en un modelo dinámico en la observación y análisis de ciertos atributos de los individuos como actores singulares y en grupo. A partir de su identificación en el sistema de relaciones, se construye un entorno particular y se articula en un contexto histórico. Se exploran asimismo los componentes de ese sistema de relaciones de la familia integrada por actores dinámicos y visionarios que sirven de una malla contenedora de su parentela y de un entramado agresivo en el mundo de los negocios y la empresa. El campo de la historia es el primero al que es sometido este modelo, con esto pretendemos analizar las características individuales de un personaje representativo de la élite queretana de mediados del siglo XVIII, para poder transitar a las acciones enmarcadas en el seno familiar y obtener algunas respuestas sobre el funcionamiento de un segmento del sistema social novohispano. El segundo campo es desde la antropología, con una aproximación particularizada en dos actores de una familia empresarial que ha logrado mantenerse vigente en el espectro económico local desde finales del siglo XIX. En este sentido, el centro de atención está puesto en la identificación de sus vínculos y mecanismos para develar las interacciones de su tejido social.

En este esquema interpretativo, es posible adelantar que ponemos especial énfasis en el carácter cualitativo de las redes, en esta ocasión no retomaremos un método cuantitativo. Además de los recursos teóricos que incluimos en el artículo, el trabajo proviene de herramientas metodológicas distintas, afín con las respectivas disciplinas, en el caso de la historia la reconstrucción del quehacer individual y familiar del actor 
seleccionado proviene de dos fondos, del Archivo Histórico del Estado de Querétaro, se seleccionaron protocolos de distintos notarios que ejercieron su oficio entre 1765 y 1820, esta información dio luz sobre los testamentos, avalúos, formación de sociedades, compras, ventas, arrendamientos y fianzas, con los que hemos podido interpretar los vínculos que forman la red de negocios; mientras que la segunda fuente consultada es la que se conserva en el Archivo de la Parroquia de Santiago, de donde obtuvimos con certeza los nexos familiares formados a partir de los enlaces matrimoniales, bautizos y atestiguamientos. En cuanto al trabajo antropológico, dado que el estudio parte del análisis de una familia y sus segmentos en el contexto actual, la información proviene de entrevistas a profundidad, visitas intensas, documentos impresos que conservan en el archivo familiar y de la empresa, así como la convivencia en el quehacer cotidiano con los directivos y los operarios; la observación participante, el trabajo de campo y el empleo de la etnografía arrojaron algunos datos que aquí presentamos. Estos pasos de investigación fueron necesarios para conocer el contexto social local y el entramado de vínculos familiares y de negocios, así como la articulación de los miembros con otros giros industriales.

Evidentemente, temporal y socialmente son casos distintos. Cada uno posee complejidades particulares, sin embargo, parece pertinente concentrar los esfuerzos en identificar los atributos más significativos de cada tema de estudio y que fueran susceptibles de ser comparados. Los hallazgos de los datos encontrados en los archivos y en el trabajo de campo fueron sometidos a una serie de discusiones académicas, este ejercicio dio cabida a la construcción de un análisis interdisciplinario, que dio como resultado el establecimiento de las propuestas que expondremos en el desarrollo del artículo. El lector quizá encontrará algunas vetas no exploradas o explicadas de manera parcial, lo mismo que la rotundidad de algunas ideas sobre el eje de lo que se estudia desde cada disciplina, de suerte que es solamente una señal que apunta la complejidad del esfuerzo por compartir y conjugar nuestros campos de trabajo.

\section{Redes sociales en historia}

Para el historiador las formas de articulación y organización social cobran relevancia en la medida que pasan de ser "objeto", a ser "sujeto" de la historia. Asimismo, la familia deja de ser un "ítem", para convertirse en la unidad central de análisis, ya que a través de las relaciones familiares es posible observar "la organización básica de los actores sociales y del ordenamiento de la producción, el trabajo, el mercado, la constitución 
política, la vida social [la familia] vincula a los actores sociales en dinámicas y procesos históricos" (Imízcoz, 2001:23). La riqueza de esta forma de análisis no debe pasarse por alto, ya que en vez de interpretaciones macrosociales, la multiplicidad de vínculos minúsculos entre un par, o varios actores induce a formular hitos que permiten entender las relaciones que se producen en cualquier sociedad y tiempo. Michel Bertrand (1999) trabaja sobre estos lineamientos, pero señala que la metodología se debe ceñir a la identificación y estudio tanto de individuos como de conjuntos sociales con ciertas características de homogeneidad, que los identifican entre sí y dan coherencia al grupo, según el autor, se trata de un grupo establecido a priori, individuos que pueden encajar en forma relativamente sencilla en un rompecabezas de variables presupuestas.

En este sentido, partimos de la consideración de revitalizar los testimonios particulares de hombres, mujeres, familia y parientes, en función de sus vínculos, ya que consideramos que de esta observación surge una forma la comprensión de la sociedad, que de ninguna manera planteamos como única, pero sí como una las más adecuadas. Es decir, la reconstrucción histórica de las acciones individuales permite identificar a los miembros de una red a partir del momento que ésta entra en acción, en este contexto y sin descartar el análisis de las estructuras, es conveniente analizar las prácticas y los vínculos de los individuos para poder descifrar el contexto social. El análisis de las estrategias personales y familiares permite observar cómo se relacionaron los individuos y cuáles fueron los enlaces que utilizaron.

La unidad social ontológica más simple, señala Georg Simmel (1950), no puede ser un individuo aislado, sino las relaciones que establece por lo menos con otro individuo, ya que para entender la sociedad la mayor fuerza del interés debe estar puesto en las interacciones que se vuelven concretas cuando los actores sociales intercambian bienes y servicios que consideran importantes es decir, las formas de relación pueden existir en forma micro, entre dos individuos como mínimo, por ejemplo en las relaciones personales, el compadrazgo, o en forma macro, mencionamos como muestra los vínculos que se observan en la composición de las familias, los estados o en las organizaciones.

Con este principio en el que las redes sociales están basadas en el intercambio de bienes y servicios formulamos la pregunta sobre ¿cómo se benefician los actores?, dicho de otra forma, ¿cómo se establece la relación costo-beneficio? Para George C. Homans (1974), quien obtiene el beneficio es el actor que paga el costo; mientras que 
para Peter Michel Blau (1994), el beneficio de un individuo implica costos para su comunidad. Creemos que para determinar quién y cómo se beneficia una relación, se deben tomar en consideración varias tramas. Sin duda alguna, el contexto para definir los diferentes tipos de relaciones sociales analizado ampliamente por los estudiosos del tema es vasto, asunto que rebasa nuestra intención, más bien apuntamos solamente aquéllos componentes afín a las dos disciplinas sociales señaladas en un principio, en este sentido, delimitamos nuestro campo de trabajo a las relaciones familiares y a las personalizadas. 2

Con esta restricción hay que reconocer en primer lugar de qué tipo de actores se trata; en segundo, la clase de relación que existe entre los actores; en tercero, la condición del nexo respecto del cual se está tratando de determinar quién y cómo se beneficia; y en cuarto lugar, hay que tomar en consideración el tipo de contexto en el que se da la relación.

Al tratar de demarcar la forma de las relaciones sociales partimos de cuatro propuestas:

1. Las relaciones personales son de naturaleza diferente respecto de las relaciones sociales.

2. Las atribuciones individuales representan una gran fuerza que moldea las relaciones, que se reflejan en forma nítida en las obligaciones familiares.

3. Es posible encontrar diversas conexiones en cada uno de los nodos de los sujetos de las redes, tal como se observa en los intereses económicos dados en las relaciones familiares, pero también se pueden encontrar en los lazos afectivos.

4. En la vida cotidiana los vínculos personales contienen rasgos que no son determinados desde su interior, ya que provienen de los contextos sociales en los que se enmarcan.

\section{Redes sociales en antropología}

Los estudios sobre redes sociales comenzaron a hacerse presentes a partir de mediados del siglo pasado, y fueron abordados desde varios ámbitos. En la década de 1960 la propuesta de Max Gluckman, de la Ilamada Escuela de Manchester, cobró gran

2 En contraposición, las redes económicas no necesariamente son de largo plazo, las más de las veces son fugaces, por lo menos en el caso de las cesiones de poderes para representaciones y cobranzas, tienen una temporalidad, que no necesariamente implica afecto. Lo que sí se involucran este tipo de relaciones es la confianza, que de igual manera es un valor entendido, difícil de cuantificar. 
relevancia, ya que dio lugar al campo de la sociometría, cuando se realizaron los estudios urbanos en Zambia. El planteamiento pretendía cuantificar las relaciones sociales en el que, para explicar una estructura social, como señala Radcliffe-Brown, hay que describirla a partir de los modelos de conducta a los que están ajustados mutuamente los individuos y los grupos. Ese acomodo tiene que ver con los intereses que cada uno presenta, ya sea por lograr una convergencia o por establecer una limitación en un juego donde puede ser objeto o sujeto de la relación establecida (Radcliffe-Brown, 1969:226-227).

La perspectiva analítica ha evolucionado de tal forma que no necesita ya de elementos justificatorios para su utilización. Las redes sociales han cobrado una enorme importancia en las ciencias sociales, de tal forma que han alcanzado distintos niveles de aplicación y uso. Por ejemplo, han sido puestas en una tipología para ubicarlas y usarlas de forma más dinámica, como la que Florence Rosemberg señala en su introducción al planteamiento de etnografía en ciudades perdidas. De la teoría sobre redes que analiza se pueden destacar los siguientes puntos de acuerdo a la referencia que hace de Barnes (1954) y Bott (1955), subraya que la red dispersa se caracteriza por las escasas relaciones entre sus actores o unidades componentes; la red conectada es en la que surgen varias relaciones y las conexiones son más dinámicas en el tiempo; la red extensa se caracteriza porque los nodos de conexión están concentrados en un actor social (ego), y es a partir de éste que se extienden las relaciones sin que los demás actores estén conectados entre si; la red efectiva es la que determina que los actores sociales tienen relaciones entre el ego, y su relación con los demás actores sociales, aunque la conexión de los nodos sea de forma horizontal y de cadena. Como vemos, cada una tiene una delimitación y articulación que la distingue de manera particular, en la que podemos encontrar una serie de aplicaciones tanto en la vida cotidiana de los sujetos, como en la teorización de los problemas de articulación de grupos sociales. Por eso nos parece pertinente destacar la reflexión que formula Florence Rosemberg en tanto que:

Las redes que se estudian desvinculándolas del mundo, de la historia, de la vida y de la naturaleza pierden su sentido explicativo y de conocimiento. La trama, el tejido de estas redes está imbricada en diversos bucles de socialización. Las redes son interacciones que están y se viven en constantes y muy diversos flujos de conectividad: es lo social conectado, engranado al mundo, a la Phycis, al oikos y a la vida de todos los vivos de este momento preciso. Pensar en redes es enredarse en la complejidad. Pensar en redes es abrir-se a la multiplicidad y a la diversidad (Rosemberg, 2002: 124). 
Bajo estos argumentos indicamos que una persona no puede sustraerse de las relaciones sociales, ya que todo individuo necesita articularse con sus semejantes para que las acciones del sujeto sean a la vez únicas y diversas en la vida social. Ante este argumento, el análisis de los vínculos se ha convertido en una herramienta pertinente para los estudios sociales de corte histórico y antropológico.

En la literatura en estos campos de estudio parte de la necesidad de observar las interacciones entre sujetos para poder entender la sociedad. El material exegético que se ha producido sobre el tema es abundante, y es compleja su clasificación, pero sugerimos que para afrontar esa complejidad podemos tomar como premisa los atributos de relaciones y acciones coyunturales sociales del sujeto. Coincidimos en que es importante estudiar las relaciones que se vuelven concretas en la vida de los sujetos en la medida que se intercambian bienes y servicios a partir de las necesidades y atributos que cada actor posee como individuo, o bien, como parte de un complejo entramado social.

\section{Algunas aplicaciones de las redes sociales en el estudio de actores y familias}

Con base en los supuestos teóricos señalados, proponemos una demarcación a partir de algunos de los resultados y propuestas que se han hecho desde la historia y la antropología mexicana. Sin restar relevancia a otras contribuciones, ponemos especial énfasis en el texto de Zacarías Moutoukias (2002), porque presenta las posibilidades que ofrece el enfoque de redes ego centradas. Su trabajo está estructurado alrededor de los: "grandes personajes de las oligarquías coloniales, estas [en referencia a las redes] constituyen en realidad tejidos que articulan los lazos comerciales" (ibid, 1). Al autor le queda corta la noción de red señalada como un conjunto complejo de relaciones recíprocas en un sistema social, por ello, decide referirse a la red como un “conjunto específico de relaciones en un grupo definido de personas" (ibid).

Por su parte, el texto de Larissa Adler Lomnitz y de Marisol Pérez Lizaur (1993), fue relevante para nuestros trabajos, ya que las autoras analizan una familia de la élite de la Ciudad de México. Encontramos que entre los elementos más interesantes del ejercicio es tanto reconstrucción del parentesco, como la perspectiva macrosocial en la que se insertan sus actores y que proporciona el marco para el entendimiento de las acciones y comportamientos individuales y familiares. En la contribución de Larissa Adler Lomnitz y Marisol Pérez Lizaur, el centro de atención está puesto en los actores 
que fueron consolidando su fortuna hasta convertirse en un importante grupo empresarial.

Metodológicamente, las autoras incluyen varios niveles de análisis: la perspectiva individualista y el contexto en el que se desarrollan los sujetos en un período específico; agregan también la faceta de grupos, en la medida que éstos tienen una participación y posición dentro de la estructura social, por tanto, también toman en cuenta el análisis desde el enfoque estructuralista. Sin duda alguna los resultados de la aportación de las autoras tienen muchas virtudes, entre ellos es conveniente resaltar dos, el primero, es que describen cómo varios de los miembros de una familia pueden establecer una serie de redes empresariales interdependientes, lo que lleva al lector a comprender el entramado de relaciones individuales y políticas. La segunda, es el hecho que en ningún momento del análisis se pierde la perspectiva de las acciones individuales, familiares y de parentesco.

Por otra parte, Molina (2001), coincide en que la formación de redes sociales permite observar las relaciones específicas entre los actores sociales, pero agrega el componente de los acontecimientos, lo cual suma otra categoría. A su juicio, a partir del análisis de las tramas encontramos datos relacionales, que son vínculos específicos entre los elementos, es decir, los nodos de conectividad de los elementos y actores sociales. Asimismo, las redes existen a partir de la acción entre los pares de elementos y las relaciones entre ellos, su aproximación brinda la oportunidad de describir una estructura social (ibid, 14-18). La red entonces, es el resultado de la relación de los grupos humanos que sostienen dos o más personas con el propósito de ayudarse, realizar negocios o llevar a cabo cualquier actividad articulada con sus intereses. Los rasgos familiares, de negocios o de producción son los más comunes encontrar en las redes sociales que se efectúan entre los integrantes de la sociedad. Las características de la red que más destacan son la flexibilidad, forma de organización y la reciprocidad. Consideramos que un complemento a las contribuciones anteriores es el que realiza Manuel Castells (1997), ya que apunta que la forma de organización proporciona una configuración a la distribución de funciones y operaciones de quienes componen un grupo o integran una estructura social. Adicionalmente, incluye el término de reciprocidad referido a la forma de intercambio de elementos materiales como intangibles que llevan a cabo sus integrantes; valores, intereses, rasgos culturales, producción y ganancias, entre otros. La lógica de esta red ha modificado el modo de 
operación y los resultados de producción, experiencia, poder y cultura en nuestras sociedades.

Queremos agregar que estas redes pueden ser descubiertas por diferentes vías, en particular mediante el trabajo prosopográfico y etnográfico. La información que de éstos métodos deriva confirma que las redes sociales se enmarcan en el sistema de relaciones sociales, en las que el actor obtiene y adquiere reconocimiento $y / 0$ beneficio social, dentro y fuera de su gremio, legitimidad y poder que hace valer en su entorno, como anticiparon en su momento autores como Adams (1974) o Parsons (1986). La manera en que se van estableciendo las redes, es a partir de la actividad y del proceso de desarrollo del individuo en su grupo social, en el cartabón de los comportamientos y leyes socialmente legitimados.

Por ejemplo, los actores económicos, en particular los empresarios se han caracterizado como aquellos responsables de las operaciones de sus empresas, como individuos capaces de desempeñar el papel de dirigentes en su relación con el Estado, con su colectividad o con el aparato productivo, clientes y proveedores, con las redes económicas locales, regionales, nacionales e internacionales (Camp, 1995; Puga y Tirado, 1992; Hernández, 2004). La forma en que están organizados influye en el sector productivo del país. (Pozas y Luna, 1991; Marichal y Cerutti; 1997). Esta imagen permite prestar atención sobre el grado de dificultad que presentan las vinculaciones y la creación de redes en las organizaciones y los actores sociales, ya que las empresas en ocasiones se convierten en actores sociales. En este sentido sugerimos que la red es una estructura intrincada de sus integrantes, que da solidez a las relaciones que establecen para perseguir un objetivo común. Entonces, la red estará sustentada a partir del intercambio de información mediante sus particulares canales de comunicación, para efectuar determinadas interacciones y transacciones necesarias conforme a sus necesidades e intereses, lo que le da un significado de evolución y transformación en la medida del propio desarrollo de cada uno de sus integrantes. El "interés" es una conducta que tiene un objeto y un valor para el sujeto, en este sentido estamos hablando de la forma en que se efectúan estas relaciones sociales en las que puede haber una relación asimétrica.

En estos ejemplos puntuales de los autores emblemáticos citados, son evidentes las coincidencias en las categorías de análisis utilizadas por ambas disciplinas, cabe entonces preguntar en este particular tema ¿dónde está la línea divisoria?, o tal vez, lo más sugerente sea preguntar también ¿hay que seguir los postulados de Immanuel 
Wallerstein (1996), formulados hace más de una década, sobre la tendencia creciente hacia el desdibujamiento de las fronteras disciplinarias? Esto permite continuar con nuestro esfuerzo en el que tratamos de demostrar que la utilización de una perspectiva analítica conjunta entre la historia y la antropología, reúne los elementos suficientes, incluso desde técnicas metodológicas distintas. Por ello, el énfasis está puesto en dos casos tomados para explorar la formación de redes sociales en temporalidades distintas. El primero esta referido a un personaje distintivo de la élite queretana del virreinato tardío, Juan Antonio del Castillo y Llata, y el segundo, a un personaje atípico del gremio empresarial de San Juan del Río en su etapa de crecimiento industrial, Francisco Salas. Los datos provienen de investigaciones amplias que hemos realizado en la sociedad queretana del siglo XVIII y del siglo XX respectivamente, y han servido como tema de tesis de doctorado. ${ }^{3}$

\section{Actores y familias en el virreinato tardío mexicano}

La amplia bibliografía que existe sobre la familia da cuenta de la necesidad de transitar hacia el reconocimiento del pasado familiar para poder avanzar con certeza hacia el entendimiento de la permanencia de individuos y de grupos que han detentado el poder. Una de las aportaciones más relevantes al estudio de las élites mexicanas virreinales a partir de las familias, es el trabajo que realizó Gloria Artís Espriu. En la parte introductoria, la autora hace la siguiente aseveración: “A lo largo de tres siglos existió en la Nueva España un grupo social que logró conservar su poder económico y político, gracias a un dinamismo que le permitió adaptarse a las circunstancias cambiantes". Para la autora el pilar sustantivo de estos mecanismos fue en el núcleo familiar (1994: 11-12).

Los testimonios escritos permiten afirmar que en el Antiguo Régimen, las familias y parentelas constituían conjuntos de gran centralidad, en este contexto las relaciones familiares y de parentesco tenían un significado mucho más amplio que el actual, sobre todo porque acumulaban tanto el capital material como el cultural, relacional y simbólico de sus miembros. Los conjuntos familiares resultantes de los diversos vínculos de parentesco, podían prolongarse mediante relaciones de amistad, lealtad y de patronazgo, incluso poseer todas estas funciones la vez. La familia reafirmaba la posición del individuo frente a la sociedad, asimismo implicaba la pertenencia, lealtad y

\footnotetext{
${ }^{3}$ Carmen Imelda González Gómez, Redes familiares y económicas en Querétaro, 1765-1821, El Colegio de Michoacán, Tesis de Doctorado, Zamora (2007). Manuel Basaldúa Hernández, Los empresarios de la Compañía Industrial de San Juan del Río: antropología de la industria cerillera, Tesis de Doctorado, Universidad I beroamericana, México (2005).
} 
sumisión de sus miembros y es aquí donde empiezan a enlazarse una serie de conceptos que apoyan el análisis de las redes sociales, ya que uno de los elementos que caracteriza a la sociedad virreinal y post virreinal no es la continuidad o discontinuidad patrones individuales y familiares, sino la combinación entre ambas para constituir vínculos organizativos; las redes sociales derivadas de las relaciones familiares funcionaron como organizaciones tendientes a reproducir el orden social (público y privado), en este sentido está presente la necesidad de transitar de las acciones individuales a las estrategias familiares-colectivas. Mediante la estructura familiar fue posible que se afianzaran los intereses de sus miembros en la sociedad; la familia actuó como grupo y con la capacidad de armar redes sociales y económicas mediante matrimonios estratégicos, inversiones y participación política.

Entre los grupos sociales existentes durante el Antiguo Régimen el más fácilmente identificable -por los testimonios que hemos ubicado en el archivo histórico local- es el de los individuos y familias de la élite; ${ }^{4}$ en las historias de los poderosos, es posible encontrar respuestas sobre las crisis económicas locales y regionales, ya que consideramos que sirven como referente del comportamiento de los grupos y las redes de propietarios, funcionarios, comerciantes, incluso hasta militares, que generalmente estaban emparentados, en este sentido está presente la necesidad de transitar de las acciones individuales a las estrategias familiares-colectivas.

Del universo de individuos y familias poderosas queretanas de mediados del siglo XVIII, seleccionamos el caso del santanderino Juan Antonio del Castillo y Llata, en el que los vínculos de paisanaje, familiares y de compadrazgo jugaron un papel crucial para la trascendencia de este personaje, estas relaciones no solamente fueron producto de una decisión consciente individual, sino que estamos ciertos que respondieron a una lógica de enriquecimiento y prestigio, que a su vez, reprodujo y protegió el patrón de comportamiento social, cuyo trasfondo era mantener el statu quo de la élite en el poder.

Juan Antonio del Castillo y Llata cumplió con las características de paisanaje y de parentesco, particularidades que a mediados del siglo XVIII fueron sustantivas para poder realizar alianzas matrimoniales interesantes, en este caso, con la hija de un conquistador. Las dos características señaladas hacen suponer que desde de su llegada a la Nueva España estableció lazos de cercanía y confianza con José de Escandón y

\footnotetext{
${ }^{4}$ Por élite entendemos a ciertos sujetos que estaban en la cúspide social y económica, individuos que lograron trascender y mantenerse en la cima del poder durante varias generaciones (tres por lo menos).
} 
Helguera conde de Sierra Gorda, de modo que este asunto le alivianó el camino en varios frentes; uno, el económico, el otro, se refiere a la carrera militar, y el último (no por orden), a su acomodo matrimonial.

Este personaje llegó a Querétaro a los 20 años, esto es, próximo a 1766; probablemente por un tiempo no se avecindó en la ciudad, existen varios documentos que lo citan como "residente en el real de minas de Xichú", en la Sierra Gorda queretana, donde comenzó a concentrar sus esfuerzos militares y combinar dos frentes económicos, el comercio y la minería. Cabe señalar que el comienzo de su vida militar y productiva coincide con la recién concluida pacificación de la Sierra Gorda queretana que la historiografía atribuye a su pariente J osé de Escandón y Helguera.

Asimismo, un factor determinante en la vida económica y social de Juan Antonio del Castillo y Llata fue su matrimonio con la criolla Josefa María Modesta de Escandón y Llera, originaria de Nuevo Santander, hija del mismo J osé de Escandón y Helguera y de la criolla acaudalada María Josefa de Llera y Ballas. En este caso el matrimonio resultó ser el mecanismo principal para adquirir y mantener riqueza y posición social, así que la elección de su cónyuge resultó decisiva.

Frente a este complejo esquema de reproducción social, un complemento sustantivo de la trama es la urdimbre de los compadrazgos; mediante estas relaciones es posible observar el reforzamiento de los lazos consanguíneos o su contraparte, la formación de nuevos nexos para crear vínculos parentales. Estamos ciertos de que los compadrazgos fueron relaciones no necesariamente consanguíneas que dieron cabida a la formación de un nuevo vínculo, esto es, al parentesco espiritual que durante el virreinato tardío fue garante de la confianza, ya que es posible suponer que la elección implicó la elección y voluntad de las partes. Daniel Santilli menciona que el compadrazgo era un "parentesco ritual [que] se materializa ante la pila bautismal, los padres del ahijado eligen al padrino y se aceptan mutuamente" (2003:3). Frédérique Langue complementa la caracterización diciendo que "además de la esfera protectora de la familia, la amistad y la religión representaban los lazos más sólidos y duraderos del mundo exterior: el compadrazgo es la expresión preponderante de esos lazos" (1999: 332).

La información recabada de Juan Antonio del Castillo y Llata, permite indicar que tuvo una preferencia por combinar los vínculos familiares con los de compadrazgo, es decir, las obligaciones morales para con los hijos se realizaron entre los miembros de su red 
familiar, lo que supone que la consolidación de la autoridad doméstica fue reforzada por vínculos internos. En este caso a pesar de que los hijos que tuvo no lograron sobrevivir por mucho tiempo, en el bautizo de su segundo hijo (José Antonio), eligió como padrinos a sus primos hermanos, pero también fue compadre de cuñados y primos políticos, es posible mencionar entonces que sus relaciones dibujan un tejido de vínculos familiares y sociales.

Cabe hacer un llamado sobre la elección de sus compadres, ya que para este acto también seleccionó y fue seleccionado por oficiales militares de rangos importantes, que fueron principalmente paisanos y parientes de Juan Antonio del Castillo y Llata. Esto lleva a la reflexión sobre la formación de una incipiente colonia santanderina capaz de actuar en varios frentes estratégicos: social, económico y militar, esta combinación de intereses homogéneos les permitió consolidarse y conformar un grupo local selecto de individuos, entonces podemos interpretar que si bien hubo una tendencia a reforzar algunas relaciones endógenas, también se percibe la fuerza de los vínculos exógenos, de modo que consideramos que el dinamismo de la red de relaciones sociales que formó con individuos externos a la familia (paisanos), constituyó un tejido amplio que contribuyó en la reproducción del sistema social.

Así como en los matrimonios y compadrazgos se hemos podido identificar parte del tejido familiar y social, consideramos necesario puntualizar las relaciones que surgieron a partir de las relaciones personalizadas, con particular atención en las administraciones de herencias y los albaceazgos. Mediante la reconstrucción de estos tejidos invisibles se pueden identificar tanto la consolidación de los lazos familiares y parentales, como algunos vínculos de nueva creación fincados en la amistad y confianza. En este sentido, subyace la noción de redes sociales señalada por Michel Bertrand (1999), como un mecanismo de corte cualitativo que permite dar respuestas a fenómenos no tan evidentes, ni medibles y que posibilita el esclarecimiento del funcionamiento del tejido social del individuo, las familias y los grupos. Las relaciones que se forman con este perfil pueden detectarse mediante el análisis de los testamentos, ya que además de transmitir propiedades y obligaciones morales, los miembros de la élite queretana también fueron receptores de estos deberes. Por ejemplo, otorgar y asumir la tarea de albacea o administrador de bienes implicaba contraer compromisos éticos y morales, de modo que en la formalización del lazo van implícitos los valores fundamentales que atraviesan este tipo de relaciones, como son 
la confianza, la lealtad y la reciprocidad, ya que se trataba de un compromiso voluntario de largo aliento entre dos individuos.

En cuanto a los vínculos de albaceazgo de Juan Antonio del Castillo y Llata, el primero que se tiene noticia fue dictado por su tía política que en su testamento lo nombró principal albacea, fideicomisario, tenedor de bienes, curador ad bona y curador ad litem, de la fortuna de su hija. También fue albacea testamentario, fideicomisario y tenedor de bienes de individuos sin relación parental, pero con distinciones militares o profesionistas reconocidos. Estos encargos de ninguna manera se presentaron como nombramientos pasivos, por el contrario, los protocolos informan de esfuerzos permanentes por parte de los administradores no sólo para proteger, sino además hacer crecer la fortuna de aquéllos depositantes de la confianza, en particular el albacea se convertía en el gestor de los deseos y de las órdenes que dictaba un individuo para que se realizaran después de su muerte. Estos vínculos suponen por un lado, el reforzamiento de los lazos familiares y parentales demostradas mediante los recurrentes nombramientos de administraciones de fortunas, y por otro lado, realizar nexos con individuos que no fueron sus pares socioeconómicos, pero que de alguna manera la relación sostenía un grupo de individuos leales sujetos mediante relaciones clientelares.

Para cerrar la caracterización de un individuo representativo de la élite queretana y la formación de redes sociales, creemos conveniente mencionar una variable más, la que se refiere a las relaciones de negocios. Juan Antonio del Castillo y Llata logró diversificar su economía, de origen fue comerciante, después hacendado, minero, obrajero, prestamista y acumuló varias casas en el casco urbano de Querétaro. Su fase de actividad económica intensiva se sitúa entre 1769 y 1817, período en el que se han logrado identificar 53 relaciones de negocios con comerciantes, mineros, funcionarios del gobierno virreinal y militares de altos rangos, como lo expresamos en el siguiente cuadro:

\begin{tabular}{|c||c|c||c||}
\hline \multirow{2}{*}{ Total de vínculos } & \multicolumn{2}{|c||}{ Tipo de vínculo } & \multirow{2}{*}{ Tipo de negocio } \\
\cline { 2 - 3 } & Familiar & Externo & Comercio \\
\hline 20 & 4 & 16 & Minería \\
\hline 10 & 4 & 6 & Haciendas \\
\hline 6 & 0 & 6 & Fincas urbanas \\
\hline 8 & 0 & 8 & Préstamos \\
\hline 9
\end{tabular}

Tabla 1: Síntesis de vínculos de negocios, 1769-1817. 
Según el resumen anterior Juan Antonio del Castillo y Llata consolidó sus relaciones familiares, pero también intensificó sus negocios con individuos externos a su grupo. Estas cifras poco indican aisladas, comparativamente, el número de relaciones tejidas por Juan Antonio del Castillo y Llata supera los nexos de negocios establecidos por otros individuos poderosos locales, por ejemplo, entre 1772 y 1805, el navarro Tomás López de Ecala realizó 43 vínculos de negocios, el criollo Pedro Antonio de Septién realizó solamente 23, y el también criollo Juan Antonio Fernández de Jáuregui tendió 32 ligas económicas.

Estamos ciertos que la calidad de los vínculos es distinta, aún así podemos afirmar que además de atender las cuestiones parentales, espirituales y de negocios, las relaciones anteriores implican compromisos y reciprocidades, en este sentido Juan Antonio del Castillo y Llata tendió puentes entre sus familias, sus pares, y con personajes socialmente relevantes, servidores leales y fieles a sus intereses, capaces de refrendar en todos los ámbitos las prácticas clientelares, que reafirmaron la consolidación del actor seleccionado en el poder local. Como empresario, actuó dentro de dos marcos, uno, reforzando las relaciones familiares y personales, mientras que el otro, atendió a individuos externos, cada uno tendió un conjunto de vínculos que juntos formaron una gran malla de sostenimiento para la élite representativa y para el funcionamiento del sistema económico, que además sirvió de soporte ante el embate económico del movimiento insurgente.

\section{Actores y familias en el crecimiento industrial mexicano}

Los actores principales de esta sección son Francisco Salas Izquierdo y Dolores Báez, que formaron una familia en la década de 1880 en el estado de Veracruz. La pareja procreó un hijo llamado Saturnino, que se casó con Mercedes León García, que también radicaron Veracruz a su vez tuvieron seis hijos: José, Rosalía, Eva, María de los Ángeles, Dolores y Francisco. Saturnino y Mercedes fueron hacendados, rancheros y propietarios de minas de minerales preciosos y piedras semipreciosas. Esta familia, además de desarrollar actividad frutícola explotando la producción de la naranja y dedicarse a la minería, también incursionó temporalmente en la elaboración casera de cerillos. Pero en los primeros años de 1900, Saturnino y su familia decidieron vender todas sus propiedades veracruzanas y emigrar a la Ciudad de México, ahí compraron una casa de grandes dimensiones, en una zona cercana al centro de la ciudad. 
Empezaron ganándose la vida utilizando sus conocimientos sobre los metales, se dedicaron a la fundición y elaboración de herrajes destinados a usarse en las ruedas de carretas que tenían gran demanda en ese tiempo. Desempeñándose en ese ramo, adquirieron algunas carretas, que destinaron al uso del transporte de personas, estableciendo una especie de carretas de alquiler. Para esta familia, estas actividades económicas tuvieron relevancia en esa etapa, pero retomaron también la elaboración de cerillos.

El tipo de formación familiar, y el sostenimiento de redes de amistades y de conocidos dentro del medio económico y familiar les permitió tener contacto con empresarios de una compañía que elaboraba cerillos que se llamaba "La Central de Veracruz", ahí se familiarizaron con el proceso de la fabricación de este producto, además este hecho también les permitió mantenerse dentro del sector productivo. Existe otra versión sobre la forma que esta familia consolidó su presencia en la Ciudad de México, esto es, la evolución del negocio de las carretas abrió otro frente no experimentado, el negocio prosperó de modo tal que pudieron ofrecer sus servicios como "sitio", de modo que conocieron a mucha gente explotando las características de las redes creadas y sostenidas, y entre estos conocidos "alguien seguramente por ahí les enseño a hacer cerillos". Como quiera que hayan sido los antecedentes, la familia adquirió el conocimiento necesario para producir cerillos de manera artesanal, pero con el propósito de comercializarlos en una escala mayor.

Es evidente que se trata de una empresa manufacturera de corte familiar, en la que los nexos interfamiliares conforman la cohesión de la mano de obra, de las capacidades organizativas, y del apoyo de todos los miembros ante contingencias económicas, laborales o administrativas. Estos aspectos fueron en gran medida lo que permitieron a Saturnino y José Salas despuntar en la incipiente actividad de la industria de cerillos de la Ciudad de México, ya que en aquel entonces, en esa primera década de 1900 había varios talleres donde se elaboraban cerillos, según refieren los familiares. Y dado que estos hermanos habían adquirido la destreza para construir máquinas rudimentarias en su empresa de fundición de metales, estas habilidades ayudaron construir algunas herramientas o "máquinas" para elaborar de una manera más rápida los cerillos. Mientras tanto, fueron adquiriendo mediante la compra otros pequeños talleres de carácter artesanal de elaboración de cerillos y fósforos. Ellos emprendieron por aquel tiempo la compañía "Salas Hermanos", la fábrica "El Indio" y "El Ángel", con todas ellas formaron a finales de la década de 1910 la fábrica de cerillos y fósforos "La 
Imperial". En este contexto se sigue manteniendo la noción de redes sociales que hemos venido retomando de Michel Bertrand (1999), que señala que son un mecanismo de corte cualitativo que deja dar respuestas a fenómenos poco visibles o medibles, pero que posibilitan el funcionamiento del tejido social del individuo con sus familias, y con ella ampliarse con los grupos de tal forma que no llegue a desintegrar la red, ni a afectarla por medio de la extracción de cualquiera de sus miembros. En el estudio de caso que presentamos, la consolidación de los vínculos entre los dos hermanos fue fundamental para el desarrollo en los negocios, incluso para la incursión en otros ámbitos económicos.

Tiempo después de haber creado la compañía cerillera, en 1919 aproximadamente, la vendieron a una familia de empresarios de ascendencia sueca, que también se dedicaba a la elaboración de cerillos. Los Salas ahora con el capital obtenido de la transacción, siguiendo con ese espíritu emprendedor, pero añorando la vida provincial, decidieron trasladarse a otra ciudad, así que eligieron una que estuviera lejana de la gran ciudad, pero cercana del centro del país y de las principales rutas de distribución; tomando en consideración estas características, a mediados de 1920 se avecindaron en San Juan del Río, en el estado de Querétaro.

En la tercera década del siglo XX, San Juan del Río era una ciudad que tenía una intensa actividad agrícola, mezclada con actividades comerciales y manufactureras entre las que se podía contar la curtiduría de pieles de bovinos, la elaboración de artesanía a base de vara de árbol, tallado de piedras semipreciosas como el ópalo, y una tradición heredada del virreinato en la elaboración de géneros textiles. En este escenario económico, los cerillos (elaborados con papel encerado tenían dos cabezas cada uno) se vendían por los vendedores ambulantes en los tianguis y como en los establecimientos del mercado municipal. La forma de en que los ofertaban era en un "manojito de diez cerillos" a un costo de 2 centavos.

En esa época pocos eran los empresarios que se habían establecido en San Juan del Río, como el propio Saturnino. La forma en que llegó a la ciudad no fue de manera directa, ya que primero exploró algunas otras opciones, como la compra de un rancho. Como refiere José Antonio, nieto de Saturnino: "Tal vez en el fondo por su mismo origen ranchero les atraía tener un rancho". Saturnino Salas llegó a una de las haciendas más emblemáticas de la región, esto es, Santa Rosa Xajay, ubicada en la parte norte de San Juan del Río, a una distancia aproximada de 20 kilómetros de la cabecera municipal. En ese tiempo, el acceso era por un camino de terracería. Las 
tierras de cultivo, si bien eran de temporal, la producción rendía lo suficiente para sostener holgadamente a la finca, los propietarios y los jornaleros.

Agrega José Antonio, que Saturnino "recorrió la Hacienda y le gustó. Su interés fue más al darse cuenta de la magnifica cosecha de maíz que se levantó y por eso decidió comprarla en 1929". Al parecer pagó cinco mil pesos por las tierras, el casco de la hacienda, trojes y aperos. Lo "simpático", dice José Antonio, es que esa fue la única y espectacular cosecha que dio la hacienda, ya que por escasez de agua la producción no se dio más de la misma manera como lo fue la primera vez. Dado que no se tuvo el éxito esperado en la hacienda Santa Rosa Xajay, que se mantenían en su punto más álgido los movimientos revolucionarios, así como la situación crítica del país respecto a la tierra, los hacendados que pudieron vendieron sus propiedades, o lo que quedaba de ellas. Estos elementos de inestabilidad provocaron que Saturnino vendiera el solar para luego trasladarse a San Juan del Río. Antes de que iniciara el periodo de la afectación de las haciendas por la Reforma Agraria ya habían adquirido unas casas extramuros de la ciudad, en la parte nor-poniente. Sin olvidar la cuestión manufacturera volvieron a fundar una fábrica de cerillos. Esta fábrica fue la primera que surgió en el entorno rural de San Juan del Río, se llamó "El Imperio Central", haciendo alusión a aquella creada por los Salas en la Ciudad de México Ilamada "La Imperial". Quisieron traer a colación que son ellos los que tuvieron la idea del nombre y brindarle un sello distintivo de la Familia. Lograron esto conjugando el nombre con las referencias de las grandes fábricas que existían en ese momento.

En el año de 1930 los Salas empezaron a levantar la fábrica, la mano de obra que se ocupaba de trabajar las parcelas de temporal fue la misma que contrataron para construir la nueva empresa. Los talleres de la fábrica se empezaron a poblar con trabajadores que provenían del ámbito rural. Gran parte de los operarios que dieron inicio de las primeras tareas de la fábrica, fueron precisamente sus antiguos jornaleros, capataces, caballerangos, que procedían de los alrededores de su antigua propiedad Santa Rosa Xajay. Una de estas personas fue Tranquilino Romero Martínez, al que consideramos como el personaje más relevante, ya que colaboró con la empresa familiar tanto en la transición del perfil de trabajadores, del campo a la industria, como en captación de artesanos. Tranquilino Romero empezó a trabajar con la familia Salas cuando tenía 12 años de edad. Pronto se incorporó a los nuevos escenarios laborales. Nos cuenta un trabajador de la cerillera, Guillermo Romero, hijo de Tranquilino, que su padre "estuvo desde la construcción de las primeras bardas hasta que se levantó 
totalmente la planta, posteriormente lo contrataron como vigilante, mas conocida la actividad como "velador" de la planta, y permaneció trabajando más de 50 años en la cerillera." Este tipo de relaciones laborales con los trabajadores rurales sirvió como modelo para contratar y mantener trabajadores dentro de empresa, asimismo, podemos mencionar que las condiciones en la empresa y sociales estuvieron comandadas fundamentalmente por Francisco Salas. Resta reconocer la capacidad de este actor para tejer vínculos con individuos que no fueron precisamente sus pares económicos, por el contrario, es evidente el desfase socio-económico entre el empresario y los operarios. En este marco vuelven a jugar un papel crucial las redes, en este caso imbricadas entre lo familiar, empresarial y el servicio; por un lado, los otrora trabajadores del campo que tuvieron la oportunidad de vincularse con un empleo de corte industrial que les posibilitó operar con ciertas ventajas con respecto a lo imprevisto de las labores agrícolas y, por otro lado el empresario, que captó hacia su fuente de trabajo individuos leales, que tenían como referente cultural la figura del señorío de las haciendas. A pesar de ser una relación mercantil, evidentemente se formaron relaciones clientelares de largo aliento.

\section{Los nodos de la cerillera contemporánea: Los hermanos Salas y la fábrica}

José Antonio Salas Reséndiz nació en 1964, en la ciudad de Querétaro. Su infancia se desarrolló en San Juan del Río donde estudió la educación básica, y una parte de la educación media. Se trasladó a Querétaro para terminar esta preparación y luego cursó en la Universidad Autónoma de Querétaro la carrera de contaduría pública y la maestría en impuestos. Desde joven se incorporó a las actividades de administración de la fábrica de cerillos, y cuando terminó la licenciatura se hizo cargo de varias tareas que su padre había estado realizando. Cuando muere Francisco Salas, José Antonio quedó al frente de la fábrica como gerente general. En 1998 fue electo Presidente de la Asociación de Industriales de San Juan del Río, A. C. En este periodo de tres años colaboró juntó con otro colega de la Asociación Sabino Núñez, vicepresidente de la misma organización, en la elaboración del documento del Plan de Desarrollo Urbano de San Juan del Río. También estuvo participando en la elaboración del rescate de la zona arqueológica del cerro y barrio de La Cruz, donde también fue designado Presidente del Patronato de rescate de esa zona y barrio. Lugares que son considerados parte importante del patrimonio histórico de San Juan del Río. Dentro de la Asociación fomentó cursos de capacitación laboral junto con el Instituto de Capacitación Tecnológica del Estado de Querétaro. Participó en pláticas para resolver el problema 
del transporte de personal y trabajadores de las empresas a las comunidades. Actualmente José Antonio, en su calidad de gerente de la fábrica de cerillos es miembro de la Cámara Nacional de la Industria de Cerillos. Su dedicación mayor esta centrada en la administración de la fábrica de cerillos, y fue el encargado de realizar las gestiones para la reubicación de esa misma fábrica.

Los hijos o familiares de los empresarios, sin tener un papel predeterminado, adoptan un rol no conciente de la responsabilidad de la empresa que por lo común, pasan a sus manos en calidad de herencia, o simplemente extienden su propiedad, y que mas adelante cuando tienen conciencia de su condición y herencia aprovechan y explotan ese bagaje cultural. ${ }^{5}$ Como nos muestra la siguiente reflexión del mismo Juan José:

Yo no pensé en terminar una carrera para después estar aquí. ¿para que me metí a estudiar ingeniería? no sé. Hubiera estudiado algún idioma, por ejemplo, no porque sea más fácil o difícil, pero no es igual. Pero me dije: bueno, si voy a estar aquí voy a estudiar algo que de alguna manera me sirva. Tengo que estudiar algo que sea afín a la empresa, al proceso industrial, para que me sirva de base para innovar o modificar el proceso. Evidentemente en la escuela no me enseñaron a hacer cerillos. Eso lo aprendí aquí. Una vez que estudie mi carrera ya pude aprender a hacer cerillos de una mejor forma. Porque ya estaba familiarizado con el cerillo. Ya que termine la carrera de Ingeniería en Instrumentación y Control de Procesos, ya tenía bases mejor cimentadas para entender el proceso industrial, mejor que si hubiera estudiado otra carrera, aunque incluso con aquella que hubiera estudiado podía dar bases para solucionar algún problema.

El trabajo de campo dentro de la fábrica y observando las acciones del ingeniero Juan José Salas, podemos indicar que las innovaciones tecnológicas no son sólo aquellos grandes descubrimientos o nuevas apariciones de máquinas complejas, sino la refuncionalización de la tecnología que esta considerada en desuso o rebasada técnicamente, por ejemplo, se rehabilitaron unas rejillas de madera que habían sido usadas en las primeras fases de la mecanización de la fábrica manejada por Saturnino y José Salas Báez. En la nueva nave industrial que ahora está situada en las tierras de la comunidad de El Chaparro, la antigua maquinaria, con gran paciencia fue armada y puesta en marcha para efectuar los procesos de producción. Aquí se esta revalorando la tradición en la fabricación en términos de procesos tecnológicos adquiridos por las generaciones previas. Esta acción no significa solamente el rescate de la esfera tecnológica, sino remite al aspecto simbólico, es decir, la articulación entre la tradición y herencia familiar con las formas de producción vigentes.

\footnotetext{
${ }^{5}$ Larissa Adler Lomnitz y Marisol Pérez Lizaur (1993), reportan que la educación de los hijos de los empresarios de la élite de la Ciudad de México y de una familia judía era semejante.
} 
La Compañía Industrial Cerillera es una fábrica que tiene un peso histórico significativo en San Juan del Río. La importancia de "la cerillera", como se le conoce en esa ciudad, radica en que marcó el derrotero de la actividad industrial, tanto por lo que representó en el inicio de la transformación laboral, como por la actividad de gestión realizada por Francisco Salas León para dar un mayor impulso a la actividad empresarial. Al interior del municipio, La "cerillera" de los Salas, junto con las fábricas que conformaban el Grupo Intra (perteneciente a la familia Peralta), son referencia obligada para comprender la historia industrial de San Juan del Río en su primera etapa. Pero al exterior del municipio, incluso para algunos de los estudiosos del tema industrial de Querétaro, (Miranda, 2004; Corona, 2001; González, 2000), “Textiles Salas, S.A." sobresale en la historia de las primeras fábricas en San Juan del Río, no siendo así la cerillera.

Entre los habitantes de San Juan del Río, la Compañía Industrial Cerillera ofreció empleo a las primeras generaciones de trabajadores industriales, operarios que luego fueron diversificando su preparación conforme a las necesidades de otras empresas; también estas primeras generaciones transmitieron la cultura laboral a sus descendientes, incluso heredaron sus puestos y habilidades para aplicar en los talleres y líneas de producción. Otro de los aspectos que logramos identificar en trabajo experimental, es el hecho que la fábrica ha mantenido sus puertas abiertas para los trabajadores del campo.

Estos hallazgos empíricos encuentran cabida en el referente señalado por Barnes (1954), sobre las redes sociales que operan de muchos niveles, desde los familiares hasta a nivel de naciones, en los que juegan un papel critico y determinan el sentido de los problemas, así como el grado de metas que se proponen los individuos. Coincidimos también con su señalamiento sobre el hecho que las redes sociales posibilitan la observancia de las relaciones sociales en términos de nodos y de lazos, señalando que los atributos personales de los individuos no son tan importantes como sus interconexiones con otros actores sociales. Por otra parte, Callon (1977) ha señalado que las redes pueden presentarse como un híbrido cuando el concepto de redes se presente como una cosa compleja. La red o las redes se extienden o se reconfiguran de acuerdo a la composición de sus elementos. Por eso señala que la reconfiguración de redes opera dentro de grupos con membresía restringida", es decir, cuando cada uno de los elementos reconoce a su nodo o su intermediario como parte operativa o de interés para la composición de la red. Así, se cumplen esas dos 
condiciones de la composición de la red, las re-configuraciones locales, y la segunda que señala que cada uno de los elementos dentro de la red juega un papel activo, que interactúa con los otros componentes" (ibid, 194). Este mismo autor señala que:

(...) estas interacciones modifican y transforman los aspectos relacionados y hacen que nuevos aspectos aparezcan en la forma de proposiciones, instrumentos, habilidades, creencias y sustancias (ibid, 194).

A nuestro juicio, lo importante es resaltar las acciones de los Salas como actores sociales que han destacado en las relaciones personalizadas, con el objetivo de consolidar, crear, mantener y articular sus vínculos sociales, que inician desde la familia y se extienden hacia sus nodos más cercanos, en ocasiones mediante relaciones de negocios. De tal forma que las redes sociales se convierten en el espacio que se conforma en el seno familiar y que trasciende a la empresa, para aprovechar ciertas oportunidades en beneficio de ambas. Evidentemente, cada uno de los participantes de la red obtiene ganancias diferenciadas, que muestran una asimetría en el otorgamiento de los recursos, pero para algunos esa es su forma de recompensa cediendo una parte importante como costo de su integración de la red. La visión de la articulación social plantea una postura diferente a la que se puede encontrar en un planteamiento desde una lógica de la economía.

Finalmente, queremos señalar que las acciones emprendidas por esta generación de Salas, fueron estrategias conscientes que tendieron buscar su consolidación como un grupo familiar-empresarial, incluso con ciertos rasgos patriarcales; pero al mismo tiempo, José Antonio Salas Reséndiz, tendió puentes con los agentes económicos y sociales más relevantes de la localidad, asunto que posibilitó su reafirmación como uno de los personajes trascendentales en la historia económica de San Juan del Río.

Estos resultados confirman la pertinencia de la utilización de la perspectiva de las redes sociales, ya que permiten establecer ciertas comparaciones aún a pesar de estar enmarcadas en períodos y contextos sociales distintos. En los casos analizados desde la historia y desde la antropología, hay coincidencias en los mecanismos que los actores seleccionados utilizaron para mantenerse dentro del poder económico y social; asimismo, mediante el aparato interpretativo verificamos las estructuras que trascienden, en particular destacamos la fuerza singular que en ambos casos tuvo la familia. 


\section{A manera de conclusión}

En el desarrollo de este trabajo tratamos de demostrar cómo las redes sociales remiten a una particular explicación de las relaciones sociales, que se puede señalar como esencial para observar determinados vínculos de intercambio. Tanto los que incluyen, como los que pueden rebasar las relaciones entre dos personas, y hasta donde dé el alcance de la interacción de las personas estudiadas. A nuestro entendimiento, se trata de correspondencias que se realizan en la esfera de los bienes y servicios, de relaciones que permiten identificar también la influencia de determinados individuos, familias y grupos sociales. Una condición importante, que ha cruzado los casos que presentamos, es la confianza, una confianza implícita que persigue metas más allá de los intereses económicos.

Asimismo, parte de las deducciones de este ejercicio muestran dos núcleos centrales, el primero, indica que el papel de la familia novohispana en el tejido social era mucho más relevante que el rol de la familia actual. La inserción social de sus miembros se efectuaba mediante una serie de estructuras que constituían el entorno en el que se desarrollarían, soportado en prácticas de fidelidades, correspondencias, solidaridades y hasta de clientelismo de la que la familia era el eje principal. En este sentido planteamos que en la formalización de este tipo de lazos van implícitos dos valores fundamentales, esto es la confianza y la lealtad; en este sentido tratamos de plantear cómo es que los compromisos de largo aliento permiten incluir el término de reciprocidad como condicionante de la formación de redes sociales. En segundo lugar, hemos encontrado que la familia forma parte de las reglas sociales que ha gobernado el sistema social; las prácticas de la vida cotidiana han sido acuñadas en su seno y asumidas por cada miembro.

Por otra parte, los ejemplos que mostramos ilustran las relaciones que cada actor tejió. Las cualidades de sus conexiones les proporcionó un gran potencial, creando ramificaciones que podían extenderse, en determinados casos, desde la familia hacia los negocios y empresas, ampliando la penetración social de los protagonistas al entremezclarse y superponerse frecuentemente, con relaciones entre: socio capitalistasocio industrial, padrino-ahijado, compadre-compadre, entre otros. Los lazos interindividuales analizados permiten poner en evidencia los medios sociales en los que se inscribieron los actores; de la misma forma que los vínculos manifiestan las posiciones sociales que ocuparon y las modalidades de inserción en la sociedad. Una de las riquezas que proporciona establecer los diferentes niveles de vínculos es que es 
posible obtener un análisis amplio de relaciones horizontales y verticales, familiares, sociales y económicas convenientes que van estableciendo los protagonistas para permanecer en el grupo de poder, a través de éstas relaciones se determinan sus redes.

Finalmente, nos hallamos ante acuerdos interdisciplinares, en el que el sistema de relaciones sociales puede ser observado a través de un modelo de red basado en otras redes secundarias pero igual de importantes, en el que el lugar de cardinal y secundario esta dado por la funcionalidad de sus componentes. Donde se expresa que los actores no sólo son individuos, sino una parte importante del entramado social, su forma de expresión es a partir de una diferenciación de los lazos o los vínculos que unen los nodos de las redes de manera asimétrica, debido a los intereses de cada uno de los nodos que unen la red presenta. La mirada a las relaciones sociales con este modelo permite observar que los actores poseen otras facetas y características, incluso más trascendentales que aquellas que llevan mecánicamente a la obtención de ganancia y aplicación de la explotación del trabajo.

\section{Bibliografía}

Artís Espriu, Gloria (1994). Familia, riqueza y poder, un estudio genealógico de la oligarquía novohispana. México: Centro de Investigaciones y Estudios Superiores en Antropología Social.

Aguilar Barajas, Ismael (1993). Descentralización industrial y desarrollo regional en México. México: El Colegio de México.

Basaldúa Hernández, Manuel, Carmen Imelda González Gómez, et Al. (2001). San Juan del Río, Pasado y Presente de la industria y sus actores. Universidad Autónoma de Querétaro. México: Serie Sociales.

Bertrand, Michel (1999). "De la familia a la red de sociabilidad". Revista Mexicana de Sociología, vol. 61, n², abril-junio, 1999, México.

- (1999). “La élite colonial en la Nueva España del siglo XVIII: un planteamiento en términos de redes sociales", en Bernard Schöter y Christian Büschges (edit.), Beneméritos, aristócratas y empresarios. Identidades y estructuras sociales de las capas urbanas en América hispánica, Separata, Vervuert, Iberoamericana, Madrid. 
Bee, Robert L. (1975). Patrones y procesos. Introducción a las tácticas antropológicas para el estudio de los cambios socio-culturales. México: Logos Consorcio Editorial.

Bertelli, D y M. Moye (1997). "Un nuevo enfoque para la política industrial regional" en E. Dussel, M. Piore y C. Ruiz. Pensar Globalmente y actuar regionalmente. Hacia un nuevo paradigma industrial para el siglo XXI. México: Ediciones JUS-FES y Universidad Nacional Autónoma de México.

Blau, Peter Michel (1994). Structural Context of Opportunities. Chicago: The University of Chicago Press.

Blim, Michael (1993). Introduction: The emerging Global Factory and Anthropology, in Anthropology and the global factory. Abrahamer Rothstein and Michael Blim. USA: Bergin \& Garvey.

Bottomore. T.B. (1975). La sociología como crítica social. España: Ediciones Península.

Bueno Castellanos, Carmen (1993). Flor de andamio. Los oficios en la construcción de vivienda de la ciudad de México. Tesis de grado. Doctorado en Antropología Social. Santa Fe, México: Universidad I beroamericana.

Caravaca Inmaculada, Gema González y Rocío Silva (2005). “Innovación, redes, recursos patrimoniales y desarrollo territorial", en Revista Eure, Vol. XXXI, No. 94. pp. 5-24, Chile.

Castells, Manuel. (1981). Crisis urbana y cambio social. México: Siglo XXI Editores.

Castells, Manuel (1985). La era de la información. España: Tomo I, Siglo XXI Editores,.

Corona Treviño, Leonel. (2001). Innovación y Región. Empresas innovadoras en los corredores Industriales de Querétaro y Bajío. Universidad Autónoma de Querétaro.

Frédérique Langue (1999). Los señores de Zacatecas. Una aristocracia minera del siglo XVIII novohispano, Fondo de Cultura Económica, México.

Gauthier, H. L. (1970), "Geography, transportation, and regional development", in Economic Geographic, Vol. 46. pp. 612-619. 
González Gómez, Carmen Imelda y Lorena Osorio (2001). La industria en Querétaro, polarización y desarrollo. México: Universidad Autónoma de Querétaro-SUPAUAQ.

Gluckman, M. (1949). The sociological theories of Malinowski. Rhodes-Livingstone Papers No. 16. London: Oxford University Press.

Haber, Stephen (1989). Industria y Subdesarrollo. La industrialización de México, 1890-1940. México: Alianza Editorial.

Homans, George Caspar (1974). Elementary Forms of Social Behavior, (2nd Ed.), New York: Harcourt Brace Jovanovich.

I mízcoz, José María (2001). “Actores sociales y redes de relaciones: reflexiones para una historia global" en José María Imízcoz (dir.). Redes familiares y patronazgo, aproximación al entramado social del País Vasco y Navarra en el Antiguo Régimen (siglos XV-XIX). Universidad del País Vasco, Euskal Herrico Universitatea.

Luna, Matilde (2003). La red como mecanismo de coordinación y las redes de conocimiento, en Itinerarios del conocimiento: formas dinámicas y contenido. Un enfoque de redes. España: Anthropos-IIS-Universidad Nacional Autónoma de México..

Lomnitz, Larissa y Marisol Pérez Lizaur (1993). Una familia de la élite mexicana, parentesco, clase y cultura 1820-1890. México: Alianza Editorial.

Mitchell, J. Clyde (1974). Social Networks. Annual Review of Anthropology. Vol. 3.

Miranda Correa, Eduardo (2005). “Del Querétaro rural al industrial, 1940-1973". México: Ediciones Porrúa-Universidad Autónoma de Querétaro.

Montes de Oca Rosas, Guadalupe (1999). Surgimiento de talleres de la industria del vestido en una región rural del México central: una perspectiva antropológica. Tesis de grado. Doctorado en Antropología Social. México: Universidad Iberoamericana, Santa Fe.

Moutoukias, Zacarías (2002). Réseaux de négociants ou réseaux ego centrés: une approche méthodologique, Université de Paris 7. http://egodoc. reuveues/org/octobre2002/docsD1074112 
Nieto Ramírez, Jaime (1998). Del Hacendado al empresario. San Juan del Río, Qro. México: Universidad Autónoma de Querétaro-Universidad Marista Campus Querétaro.

Rosemberg, Florence (2002). “Redes sociales y complejidad, una etnografía en una ciudad perdida en la ciudad de México" en Antropología: estudios de medio ambiente y urbanismo, Rafael Pérez-Taylor (coord.). México: Ediciones del Instituto de Investigaciones Antropológicas, Universidad Nacional Autónoma de México.

Santilli, Daniel (2003). “Representación gráfica de redes sociales. Un método de obtención y un ejemplo histórico", en Mundo Agrario. Revista de estudios rurales, No. 6, primer semestre de 2003, Centro de Estudios Histórico Rurales, Universidad Nacional de la Plata, pp. 1-22.

Georg Simmel (1950), The sociology of Georg Simmel, compilación, traducción, edición e introducción de Kurt H. Wolff, Glencoe, The Free Press, 1908 y 1917, 445 pp.

Immanuel Wallerstein, coord., (1996). Abrir las Ciencias Sociales. México: Siglo XXIUniversidad Nacional Autónoma de México.

Wolf, Eric. R. (1989). "Relaciones de parentesco, de amistad y de patronazgo en las Sociedades complejas", en M. Banton (comp.), Antropología social de las relaciones Complejas. Madrid: Alianza. 